\title{
Transmission cycles in parasites
}

\author{
G. $\mathrm{HIDE}^{1 *}$ and A. J. TREES ${ }^{2}$ \\ ${ }^{1}$ Centre for Parasitology and Disease Research, School of Environment and Life Sciences, University of Salford, Salford, \\ M5 $4 W T, U K$ \\ ${ }^{2}$ Faculty of Veterinary Science, University of Liverpool, Liverpool, L69 7ZF
}

(Received 27 Fuly 2009; accepted 30 Fuly 2009; first published online 21 September 2009)

Parasites are masters of survival under extreme conditions. Their continued existence relies on a highly evolved ability to survive within a host and to be able to transmit effectively between hosts. As many parasites are important human and animal pathogens, parasitologists have had long-term interests in solving the problems of survival and transmission. The 2008 British Society for Parasitology (BSP) Autumn Symposium and this issue of Parasitology were conceived to explore recent data on transmission cycles in the context of parasites of medical and veterinary importance.

Two broad modes of transmission of parasites are recognised: horizontal transmission occurs from individual to individual and vertical transmission occurs from one generation of host to the next. Various parasites utilise these different modes in different ways and our ability to control the spread of parasitic diseases requires a detailed understanding of these strategies of transmission. Horizontal transmission requires a period of time outside the host body and may be mediated by means of vector organisms, alternative hosts or by environmental forces. Thus a parasite must have evolved the machinery to take advantage of these to ensure transmission. Parasites utilising vertical transmission, on the other hand, must adopt mechanisms of transmission from one generation to the next. This could result in the parasite hijacking or even controlling the physiology of its host to promote transmission. In some parasites it is possible that both horizontal and vertical modes of transmission may be employed.

Toxoplasma gondii is one such parasite that utilises both modes of transmission. It is a major pathogen of particular concern due to its role as a causative agent of miscarriage in humans and domestic animals. This

* Corresponding author: Centre for Parasitology and Disease, School of Environment and Life Sciences, University of Salford, Salford, M5 4WT, UK. Tel: 0044161-295-3371.Fax: 0044-161-295-5015.E-mail: g.hide@ salford.ac.uk parasite of the cat is found in all warm blooded animals and is very often widespread with high reported prevalences (typically 30 to $40 \%$ ). Although the principal route of transmission of this parasite is by means of infected oocysts shed by the cat, ingestion of parasite infected meat and congenital transmission contribute to the overall distribution of this parasite. The relative importance of these different transmission routes is currently under debate. Some studies in sheep, mice and humans (reviewed by Hide et al. in this special issue) have shown that congenital transmission may be a much more important route than has been previously reported. On the other hand, there is a considerable body of evidence supporting infrequent congenital transmission. This conflict is highlighted in sheep where very elegant and careful studies have demonstrated a very limited role for vertical transmission (reviewed here by Innes et al.)

Vertical transmission in the Toxoplasma-related parasite, Neospora caninum, is highly efficient and transmission during pregnancy may be its most important distribution strategy. This parasite of the dog is a key factor causing abortion in cattle by foetal infection during pregnancy. This raises interesting questions as to the mechanisms by which this parasite manipulates its way from generation to generation during pregnancy. It could occur by endogenous transplacental transmission resulting from activation of a quiescent stage during pregnancy or exogenous transplacental transmission resulting from ingestion of oocysts during pregnancy (reviewed in this special issue by Williams et al.). An understanding of these mechanisms is important in the control of this economically important parasite.

The control of the host to promote vertical transmission is exemplified in Microsporidian parasites. In some species of Microsporidia the parasite has been shown to distort the sex ratio of the invertebrate host offspring. They cause the generation of more females enabling a more efficient transmission, vertically, through the female line. This group of parasites causes disease in immunocompromised people 
but the many species are very widely distributed throughout the animal kingdom. A mixed transmission strategy, with both horizontal and vertical modes, may contribute to the wide evolutionary and ecological distribution of the parasites (see Smith in this issue).

In contrast a parasite that is primarily transmitted horizontally, Leishmania infantum, is restricted geographically by the distribution of an insect vector. It causes Zoonotic Visceral Leishmaniasis (ZVL), an important disease of humans and dogs and is maintained principally by sandfly transmission. The putative sylvatic reservoir(s) of ZVL remain unknown and the dog is the only confirmed primary reservoir of infection. Success in controlling this important disease can only be achieved by a detailed understanding of the population interactions between host reservoirs and sandflies (Quinnell et al. in this issue).

Recent studies have focused on the population and evolutionary biology of mosquito-Plasmodium interactions. 'Ecological immunology', as it has been termed, describes the processes that create or maintain variation in mosquito immune responses and parasite virulence in natural populations (Tripet et al. in this issue). Knowledge of the vector-parasite interactions in their ecological and evolutionary context is important for our understanding of malaria transmission and how to control it.
The transmission cycles involving Trypanosoma brucei, the tsetse fly and its hosts appear straightforward. However, attempts to control human sleeping sickness and animal trypanosomiasis have eluded us for over 100 years. Detailed studies of host-tsetse interactions, distribution of parasite strains and population dynamics of hosts have recently led to the promise of sustainable control programmes for both human and animal disease. Public-private partnerships have been set up to develop simple, cost effective tools and strategies for sustainable sleeping sickness control and surveillance (reviewed by Welburn et al. in this issue).

The effective control of parasitic diseases is dependent on a clear and detailed understanding of transmission cycles. This will develop from both focused applied research on control and from curiosity-driven research on parasite-derived interactions.

We would like to thank the sponsors of the BSP Autumn Symposium, Cambridge University Press and the British Society for Parasitology, for generous support. We would also like to thank the speakers for their presentations and for the contributed papers in this special issue. We would also like to acknowledge the unseen work of the external referees and editors whose efforts have undoubtedly improved the quality of the review papers published in this special issue of Parasitology. 